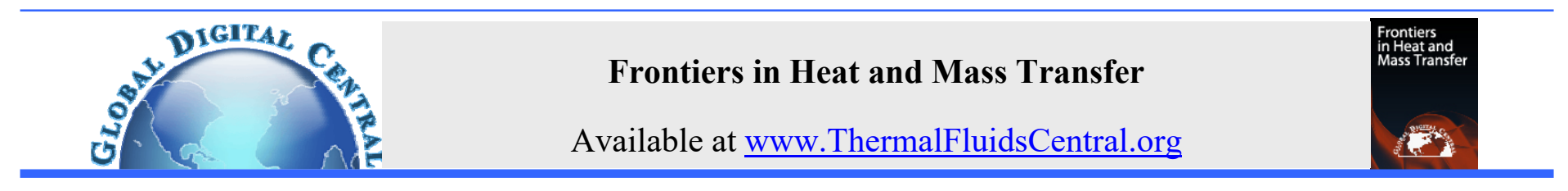

\title{
UNSTEADY MHD FREE CONVECTION JEFFERY FLUID FLOW OF RADIATING AND REACTING PAST A VERTICAL POROUS PLATE IN SLIP-FLOW REGIME WITH HEAT SOURCE
}

\author{
K. Venkateswara Raju ${ }^{a^{*}}$, A. Parandhama ${ }^{\mathrm{a}}$, M.C. Raju ${ }^{\mathrm{b}}$, K. Ramesh Babu \\ ${ }^{a}$ Department of BS\&H., Sree Vidyanikethan Engineering College (Autonomous), A. Rangampet, Tirupati, A.P., 517103, India. \\ ${ }^{b}$ Department of Mathematics, Annamacharya Institute of Technology and Sciences (Autonomous), Rajampet, A.P., 516126., India.
}

\begin{abstract}
This manuscript presents an analytical investigation on unsteady MHD free convective viscous incompressible flow of electrically conducting Jeffery fluid with heat source, past an infinite vertical porous flat plate in slip flow regime. A uniform magnetic field perpendicular to the plate is applied. The presence of thermal radiation, heat source, radiation absorption and chemical reaction are included. The effects of flow parameters and thermo physical properties on the velocity temperature and concentration fields across the boundary layer are investigated. The forms of the wall Shear stress, Nusselt number and Sherwood number are derived. The results are shown in figures and tables followed by a quantitative discussion.

Keywords: Chemical reaction, Heat source, Jeffery fluid, MHD and Slip-flow regime.
\end{abstract}

\section{INTRODUCTION}

Non-Newtonian transport phenomena arise in many branches of engineering such as mechanical, chemical and materials engineering. Such fluids exhibit shear strain relationships which diverge significantly from the classical Newtonian (Navier Stokes) model. Most non-Newtonian models involve some form of modification to the momentum conservation equations. These include Power-law fluids, Visco-elastic fluid model, Walters-B short memory models, Oldroyd-B models, differential Reiner-Rivlin models, Bingham plastics, tangent hyperbolic models, Eyring-Powell models, nano fluid models and Maxwell fluid models. Among the several non-Newtonian models proposed, Jeffrey fluid model is one of the significant models, because Newtonian fluid model can be deduced from this as a special case by taking $\lambda_{1}=0$. Further, it is speculated that the physiological fluids such as blood exhibit Newtonian and non-Newtonian behaviors during circulation in a living body. As with a number of rheological models developed, the Jeffrey's model has proved quite successful. This simple, yet elegant rheological model was introduced originally to simulate earth crustal flow problems (1929). This model (1987) constitutes a Visco-elastic fluid model, which exhibits shear thinning characteristics, yield stress and high shear viscosity. The Jeffrey fluid model degenerates to a Newtonian fluid at a very high wall shear stress, when the wall stress is much greater than yield stress. This fluid model approximates reasonably well the rheological behavior of other liquids including physiological suspensions, foams, geological materials, cosmetics, syrups and also this model finds applications in metallurgical materials processing, Chemical Engineering flow control, etc.

In many practical applications, the particle adjacent to a solid surface no longer takes the velocity of the surface. The particle at the surface has a finite tangential velocity; it "slips" along the surface. The flow regime is called the slip -flow regime and this effect cannot be neglected. To describe the phenomenon of slip, Navier introduced a boundary condition, which states that the component of the fluid velocity tangential to the boundary walls is proportional to tangential stress. Rudraiah et al. (1988) studied the effects of slip velocity and transverse magnetic field on an electrically conducting viscous fluid in a horizontal channel bounded on both sides by porous substrates of finite thickness which is analogous to the problem of forced convection where the momentum equation is independent of concentration distribution and the diffusion equation is coupled with the velocity distribution using B J slip condition at the porous interface. Later, several researchers extended the Navier boundary conditions. Martin and Boyd (2001) analyzed Blasius boundary layer problem in the presence of slip boundary condition. Investigators restricted their analyses to the flow and heat transfer with no-slip boundary condition. But, as with most engineering approximations, the no-slip condition does not always hold in reality. Investigation shows that slip flow happens when the characteristic size of the flow system is small or the flow pressure is very low. Numerous investigations have been done analytically and numerically regarding the slip flow regime.

Magnetohydrodynamic (MHD) flows also have many important technological and industrial applications including smart lubrication systems, separation devices, biometric pumps, magnetic blood pumps, and nano materials fabrication. The application of magnetic fields in electrically conducting flows generates Lorentz forces, which are able to control the flow characteristics. At high intensities Hall currents and other phenomena may also arise, including magnetic induction, Maxwell displacement currents, Ohmic dissipation, etc. Many flow regimes have been investigate using a diverse array of numerical methods. Many studies have done to understand its characteristics in different systems such as reciprocating engines, pulse combustors and chemical reactors, etc. MHD transient free convection with slip flows is associated with many applications in heat and mass transfer. The energy flux caused by a composition gradient is called the Dufour or diffusion -thermo effect. Temperature gradients can also create mass fluxes, and

\footnotetext{
${ }^{*}$ Corresponding author, Email: venky.sakku@gmail.com
} 
this is the Soret or thermal diffusion effect. The Soret effect, for instance, has utilized for isotope separation, and in mixtures between gases with very light molecular weight.

In all these investigations, analytical or numerical solution are obtained assuming conditions for fluid velocity and temperature at the plate as continuous and well defined. Keeping in view this fact, several researchers investigated free convection flow from a vertical porous plate with variable suction like Kim et al. (2000), Sharma and Chaudhary (2003), Mittal and Bijalwan (2005), Rajput et al. (2008), Pal and Talukdar (2010), Devi and Raj (2011). In all the above-mentioned investigations, it is noted however that they have not considered slip flow due to jump in concentration which is practically important. Convective heat transfer and entropy generation analysis on Newtonian and non-Newtonian fluid flows under slip boundary conditions was studied by Shojaeian and Kosar (2014).

Vajravelu et al. (2008) presented the influence of free convection on nonlinear peristaltic transport of Jeffrey fluid in a finite vertical porous stratum using the Brinkman model. Radiative flow of Jeffery fluid in a porous medium with Power law heat flux and heat source is studied Hayat et al. (2012). Many numerical and analytical investigations on Jeffrey fluid have been carried out by several researchers. In another investigation, Hayat et al. (2012) considered similar effects of thermal radiation and porous medium to examine the flow of an incompressible Jeffrey fluid over a stretching surface together with Power-law heat flux and heat source. Shehzad et al. (2013) investigated the influence of thermophoresis and joule heating on the radiative flow of Jeffrey fluid with mixed convection. Idowu et al. (16) proposed the effect of heat and mass transfer on unsteady MHD oscillatory flow of Jeffrey fluid in the horizontal channel with chemical reaction. Ahmed and Kishor Kumar Das (2013) presented the effects of thermal radiation and chemical reaction on MHD unsteady mass transfer flow past a semi-infinite vertical porous plate embedded in a porous medium in a slip flow regime with variable suction. Meanwhile, a comprehensive discussion of thermal radiation to investigate the hydromagnetic flow of an incompressible Jeffrey nanofluid over an exponentially stretching surface is reported by Hussain et al. (2014). They also considered the viscous dissipation, Brownian motion and also thermophoresis effects in their study.

Nallapu and Radhakrishnamacharya (2014) studied Jeffrey fluid flow in the presence of magnetic field through porous medium in tubes of small diameters. Sreenadh et al. (2014) investigated unsteady flow of a conducting Jeffrey fluid in a horizontal composite porous medium channel and it is found that the velocity decreases with the increase in the non-Newtonian Jeffrey parameter whereas the temperature shows same trend with the Jeffrey parameter. For a given ratio of viscosity, the interface velocity decreases with increasing magnetic parameter and porous medium parameter. The influence of slip and heat transfer on the peristatic transport of Jeffrey fluid in a vertical asymmetric channel in porous medium is discussed by Lakshminarayana et al. (2015). The influence of melting heat transfer and thermal radiation on MHD stagnation point flow of an electrically conducting Jeffrey fluid over a stretching sheet with partial surface slip is performed by Das et al. (2015). Sreenadh et al. (2016) investigated free convective flow of a Jeffrey fluid in a vertical deformable porous stratum. They observed that the skin friction gets reduced when the porous material is a deformable one and noticed that the effect of increasing Jeffrey parameter is to increase the skin friction in the deformable porous stratum.

Numerous investigations has been done by different technique numerically regarding non-Newtonian fluid flows through porous medium; Khan (2007) used fractional calculus approach and analyzed the partial slip effects on the oscillatory flows of a fractional Jeffrey fluid in a porous medium via Fourier transform. Hayat et al. (2010) implemented the fractional calculus and Fourier transform technique to investigate the problem of oscillatory rotating flows of a fractional Jeffrey fluid filling a porous space. Amongst them, Hayat et al. (2010) investigated the effect of thermal radiation on the unsteady mixed convection flow of a Jeffrey fluid past a porous vertical stretching surface using Homotopy analysis method (HAM). Steady flows of a Jeffrey fluid for stretching/shrinking sheet were also investigated. Shehzad et al. (2014) illustrated their problem by considering the MHD three-dimensional flow of Jeffrey fluid with Newtonian heating and solved it by using HAM. Recently, Khan (2017) used Laplace transform technique and studied the unsteady free convection flow of a Jeffrey fluid past a vertical plate. Recently, the effect of unsteady MHD free convective flow of a Visco-elastic incompressible electrically conducting fluid past a moving vertical plate through a porous medium with time dependent oscillatory permeability and suction in presence of a uniform transverse magnetic field and heat source and chemical reaction along with heat and mass transfer are reported by Ramesh et al. (2017).

The objective of this paper is to analyze the effect of Hall Current on MHD slip flow along an infinite vertical porous plate with Soret effect and radiation in rotating system. The aim of the present study is an extension to extending the work of Sing and Kumar (2011) by including the effects of Jeffrey parameter on heat source Hall current in rotation frame of reference.

\section{FORMULATION OF THE PROBLEM}

We consider an unsteady MHD free convective flow of an incompressible, electrically conducting, thermally radiating and chemically reacting Jeffrey fluid past an infinite vertical porous plate in slip-flow regime with periodic temperature and concentration. The suction velocity is assumed to be of the form

$$
v^{*}=-v_{0}^{*}\left(1+\in \mathrm{Ae}^{i \omega^{*} t^{*}}\right)
$$

where $v_{0}{ }^{*}$ is the mean suction velocity, $\omega^{*}$ is the frequency of oscillation, $t^{*}$ is the time, A is a positive real constant and $\in$ is small such that $A \in<<1$. We introduce a co-ordinate system with wall lying vertically in $x^{*}$ and $y^{*}$ plane. The $x^{*}$ - axis is taken in vertical upward direction along the vertical porous plate and $y^{*}$ - axis is taken normal to the plate. A magnetic field of uniform strength $\mathrm{B}_{0}$ is introduced normal to the plate. Since the plate is considered infinite in $x^{*}$ - direction, hence all physical quantities are independent of $x^{*}$ and therefore functions of $y^{*}$ and $t^{*}$ only.

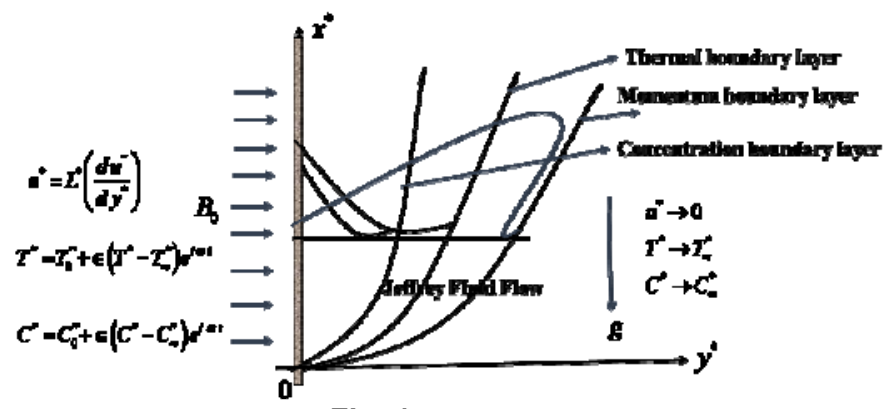

Fig. 1 Physics model

We further assume that

1. The magnetic Reynolds number is small so that the induced magnetic field is negligible in comparison to the applied magnetic field.

2. The fluid is considered to be gray: absorbing - emitting radiation but a non-scattering medium.

3. Viscous dissipation and Joule heating terms are neglected as small velocity usually encountered in free convection flows.

4. No external electric field is applied and effect of polarization of ionized fluid is negligible, therefore electric field is assumed to be zero.

5. There exists a first order chemical reaction between the fluid and species concentration.

6. The level of species concentration is very low so that the heat generated during chemical reaction can be neglected. 
Using the Boussineq's and boundary layer approximation, the governing equations for this problem can be written as follows.

$$
\begin{aligned}
& \frac{\partial u^{*}}{\partial t^{*}}-v_{0}^{*}\left(1+\in A e^{i \omega^{*} t^{*}}\right) \frac{\partial u^{*}}{\partial y^{*}}=\frac{v}{1+\lambda_{1}} \frac{\partial^{2} u^{*}}{\partial y^{* 2}}+g \beta\left(T^{*}-T_{\infty}^{*}\right)+g \beta_{c}\left(C^{*}-C_{\infty}{ }^{*}\right) \\
& -\frac{\sigma B_{0}^{2}}{\rho} u-\frac{v}{K_{2}} u^{*} \\
& \rho C_{p}\left(\frac{\partial T^{*}}{\partial t^{*}}-v_{0}^{*}\left(1+\in A e^{i w^{*} t^{*}}\right) \frac{\partial T^{*}}{\partial y^{*}}\right)=k \frac{\partial^{2} T^{*}}{\partial y^{* 2}}-\frac{\partial q_{r}^{*}}{\partial y^{*}} \\
& -\mathrm{Q}_{0}^{*}\left(\mathrm{~T}^{*}-\mathrm{T}_{\infty}\right)+\mathrm{Q}_{1}^{*}\left(\mathrm{C}-\mathrm{C}_{\infty}\right), \\
& \frac{\partial C^{*}}{\partial t^{*}}-v_{0}^{*}\left(1+\in A e^{i w^{*} t^{*}}\right) \frac{\partial C^{*}}{\partial y^{*}}=D \frac{\partial^{2} C^{*}}{\partial y^{* 2}}-K_{1}\left(C^{*}-C_{\infty}^{*}\right)
\end{aligned}
$$

where $\lambda_{1}, g, T^{*}, C^{*}, B_{0}, D, \sigma, k, C_{p}, v, \rho, \beta, \beta_{c}, q^{*}, K_{1}$ are Jeffrey fluid parameter, acceleration due to gravity, fluid temperature, species concentration, magnetic field, chemical molecular diffusivity, electrical conductivity, thermal conductivity, and specific heat at constant pressure, kinematic viscosity, density, coefficient of volume expansion with species concentration, radiative heat flux and chemical reaction parameter, respectively. Under the above assumptions, the appropriate boundary conditions for the distributions of velocity, temperature and concentration are given by

$\left.\begin{array}{l}u^{*}=L^{*}\left(\frac{\partial u^{*}}{\partial y^{*}}\right), \quad T^{*}=T_{0}^{*}+\in\left(T^{*}-T_{\infty}^{*}\right) e^{i \omega^{*} t^{*}}, \\ C^{*}=C_{0}^{*}+\in\left(C_{0}^{*}-C_{\infty}^{*}\right) e^{i \omega^{* *}}\end{array}\right\}$ at $y=0$

$u^{*} \rightarrow 0, T^{*} \rightarrow T_{\infty}^{*}, C^{*} \rightarrow C_{\infty}^{*}$ at $y^{*} \rightarrow \infty$

where $T_{0}{ }^{*}$ and $T_{\infty}{ }^{*}$ are the temperature at the wall and at infinity, $C_{0}{ }^{*}$ and $C_{\infty}{ }^{*}$ are the species concentration at the wall and at infinity respectively.

The local radiant for the case of an optically thin gray gas as expressed by

$$
\frac{\partial q_{r}^{*}}{\partial y^{*}}=-4 a^{*} \sigma^{*}\left(T_{\infty}^{*^{*}}-T^{* 4}\right)
$$

where $a^{*}$ is the mean absorption coefficient and $\sigma^{*}$ is Stefan-Boltzmann constant, We assume that the temperature differences within the flow are sufficiently small such that $T^{* 4}$ may be expressed as a linear function of the temperature. This is accomplished by expanding $T^{* 4}$ in a Taylor series about $T_{\infty}^{*}$ and neglecting higher - order terms, thus

$T^{*^{*}} \cong 4 T_{\infty}^{*^{3}} T^{*}-3 T_{\infty}^{*}$

By using Esq. (6) and (7) Eq. (2) reduces to

$\rho C_{p}\left(\frac{\partial T^{*}}{\partial t^{*}}-v_{0}^{*}\left(1+\in A e^{i \omega i}\right) \frac{\partial T^{*}}{\partial y^{*}}\right)=k \frac{\partial^{2} T^{*}}{\partial y^{* 2}}-16 a^{*} \sigma^{*} T^{* 3}{ }_{\infty}\left(T^{*}-T_{\infty}^{*}\right)$,

Introducing following non-dimensional parameters

$\operatorname{Pr}=\frac{\mu C_{p}}{k}($ Prandtl number $) ; S c=\frac{v}{D}($ Schmidt number $) ; h=\frac{v_{0}^{*} L^{*}}{v}($ Slip Parameter $)$

$G r=\frac{v g \beta\left(T_{0}^{*}-T_{\infty}^{*}\right)}{v_{0}^{*}}\left(\right.$ Thermal Grashof number); $M=\frac{B_{0}}{v_{0}^{*}} \sqrt{\frac{\sigma v}{\rho}}$ (Hattman number);

$G m=\frac{v g \beta_{c}\left(\mathrm{C}_{0}{ }^{*}-C_{\infty}^{*}\right)}{v_{0}^{*}}$ (Mass Grashof number),

$R=\frac{16 a^{*} v^{2} \sigma^{*} T_{\infty}^{*^{*}}}{k v_{0}^{*}}\left(\begin{array}{l}\text { Radiation } \\ \text { parameter }\end{array}\right) \quad \gamma=\frac{K_{1} v}{v_{0}^{*}}$ (Chemical reaction parameter $)$,

$u=\frac{u^{*}}{v_{0}{ }^{*}} ; \theta=\frac{T^{*}-T_{\infty}^{*}}{T_{0}^{*}-T_{\infty}^{*}} ; \phi=\frac{C^{*}-C_{\infty}^{*}}{C_{0}^{*}-C_{\infty}^{*}} ; \quad y=\frac{y^{*} v_{0}^{*}}{v} ; t=\frac{t^{*} v_{0}^{* 2}}{4 v} ; \omega=\frac{4 v \omega^{*}}{v_{0}^{* 2}}$

into the equations (1), (3) and (8), we get $\frac{1}{4} \frac{\partial u}{\partial t}-\left(1+\in \mathrm{Ae}^{i \omega t}\right) \frac{\partial u}{\partial y}=\frac{1}{\left(1+\lambda_{1}\right)} \frac{\partial^{2} u}{\partial y^{2}}+G_{r} \theta+G_{m} \phi-M^{2} u-\frac{1}{K_{2}} \mathrm{u}$

$\frac{1}{4} \frac{\partial \theta}{\partial t}-\left(1+\in \mathrm{Ae}^{i \omega t}\right) \frac{\partial \theta}{\partial y}=\frac{1}{P_{r}} \frac{\partial^{2} \theta}{\partial y^{2}}-\left(\frac{R}{P_{r}}+Q_{0}\right) \theta+\mathrm{Q}_{1} \phi$

$\frac{1}{4} \frac{\partial \phi}{\partial t}-\left(1+\in \mathrm{Ae}^{i \omega t}\right) \frac{\partial \phi}{\partial y}=\frac{1}{S_{c}} \frac{\partial^{2} \phi}{\partial y^{2}}-\gamma \phi$

The boundary conditions to the problem in dimensionless form are

$u=h \frac{\partial u}{\partial y}, \theta=1+\epsilon e^{i \omega t}, \phi=1+\epsilon e^{i \omega t}$ at $y \rightarrow 0$

$u \rightarrow 0, \quad \theta \rightarrow 0, \quad \phi \rightarrow 0 \quad$ at $y \rightarrow \infty$

\section{SOLUTION OF THE PROBLEM}

The set of Eqs. (10) to (12) are partial differential equations which cannot be solved in closed form. However, these can be solved by reducing them into a set of ordinary differential equations using the following perturbation technique. We now represent the velocity, temperature and concentration distributions in terms of harmonic and non-harmonic functions as

$$
\begin{aligned}
& u=u_{0}(y)+\varepsilon e^{i \omega t} u_{1}(y)+O\left(\varepsilon^{2}\right)+\ldots \ldots \ldots \\
& \theta=\theta_{0}(y)+\varepsilon e^{i \omega t} \theta_{1}(y)+O\left(\varepsilon^{2}\right)+\ldots \ldots \ldots \\
& C=C_{0}(y)+\varepsilon e^{i \omega t} C_{1}(y)+O\left(\varepsilon^{2}\right)+\ldots \ldots \ldots . .
\end{aligned}
$$

Substituting Eqs. (15) to (17) into Eqs.(10) to (12), assuming the small amplitude oscillations $(\in<<1)$, and equating the harmonic and nonharmonic terms and neglecting the higher order terms of $\varepsilon$, we obtain the following pairs of equations of order zero and order one we get

$$
\begin{aligned}
& \frac{d^{2} u_{1}}{d y^{2}}+\left(1+\lambda_{1}\right) \frac{d u_{1}}{d y}-\left(1+\lambda_{1}\right)\left(M^{2}+\frac{1}{K_{2}}+\frac{i \omega}{4}\right) u_{1}= \\
& -\left(1+\lambda_{1}\right)\left(\operatorname{Gr} \theta_{1}\right. \\
& \frac{d^{2} \theta_{0}}{d y^{2}}+\operatorname{Pr} \frac{d \theta_{0}}{d y}-\operatorname{Pr} \chi \theta_{0}-\left(\operatorname{Pr} Q_{0}+R\right) \theta_{0}=-\operatorname{Pr} Q_{1} \phi_{0} \\
& \frac{d^{2} \theta_{1}}{d y^{2}}+\operatorname{Pr} \frac{d \theta_{1}}{d y}-\operatorname{Pr} \chi \theta_{0}-\left(R-\operatorname{Pr} Q_{0}+\frac{i \omega \operatorname{Pr}}{4}\right) \theta_{1}=-A \operatorname{Pr} \frac{d \theta_{0}}{d y}-\mathrm{Q}_{1} \operatorname{Pr} \phi_{1} \\
& \frac{d^{2} \phi_{0}}{d y^{2}}+S c \frac{d \phi_{0}}{d y}-S_{c} \gamma \phi_{0}=0 \\
& \frac{d^{2} \phi_{1}}{d y^{2}}+S c \frac{d \phi_{1}}{d y}-S c\left(\gamma+\frac{i \omega}{4}\right) \phi_{1}=-A S c \theta_{0}^{\prime}
\end{aligned}
$$$$
\frac{d^{2} u_{0}}{d y^{2}}+\left(1+\lambda_{1}\right) \frac{d u_{0}}{d y}-\left(1+\lambda_{1}\right)\left(M^{2}+\frac{1}{K_{2}}\right) u_{0}=-\left(1+\lambda_{1}\right)\left(G r \theta_{0}+G m \phi_{0}\right)
$$$$
-\left(1+\lambda_{1}\right)\left(G r \theta_{1}+G m \phi_{1}+A \frac{d u_{0}}{d y}\right)
$$

The corresponding boundary conditions reduce to

$u_{0}=h \frac{\partial u_{0}}{\partial y} ; \quad u_{1}=h \frac{\partial u_{1}}{\partial y} ; \theta_{0}=1 ; \theta_{1}=1 ; \phi_{0}=1 ; \phi_{1}=1$, at $y=0$

$u_{0}=0 ; u_{1}=0 ; \theta_{0}=0 ; \theta_{1}=0 ; \phi_{0}=0 ; \phi_{1}=0$, at $y \rightarrow \infty$

Solving Eqs (18) to (23) under the boundary conditions (24-25), we get the following set of solutions

$$
\begin{aligned}
& u_{0}=l_{17} e^{-m_{10} y}+l_{11} e^{-m_{6} y}+\left(l_{12}+l_{13}\right) e^{-m_{2} y} \\
& \theta_{0}=\left(1-l_{3}\right) e^{-m_{6} y}+l_{3} e^{-m_{2} y} \\
& \phi_{0}=e^{-m_{2} y} \\
& u_{1}=l_{29} e^{-m_{12} y}+l_{29} e^{-m_{8} y}+\left(l_{20}+l_{24}\right) e^{-m_{2} y}+l_{21} e^{-m_{6} y}+\left(l_{22}+l_{23}\right) e^{-m_{4} y} \\
& \theta_{1}=\left(1-l_{9}\right) e^{-m_{8} y}+\left(l_{6}+l_{8}\right) e^{-m_{2} y}+l_{5} e^{-m_{6} y}+l_{7} e^{-m_{4} y} \\
& \phi_{1}=\left(1-l_{2}\right) e^{-m_{4} y}+l_{2} e^{-m_{2} y}
\end{aligned}
$$

\section{SKIN-FRICTION:}

Knowing the velocity field, the expression for the skin- friction coefficient at the plate in the $x^{*}-$ direction is given by 


$$
\begin{aligned}
C_{f x}=\left(\frac{\partial u}{\partial y}\right)_{y=0} & =\left[-m_{10} l_{17}-m_{6} l_{11}-m_{2}\left(l_{12}+l_{13}\right)\right]+ \\
& \in e^{i w t}\left[-m_{12} l_{29}-m_{8} l_{19}-m_{2}\left(l_{20}+l_{24}\right)-m_{6} l_{21}-m_{4}\left(l_{22}+l_{23}\right)\right]
\end{aligned}
$$

\section{NUSSELT NUMBER:}

From the temperature field, the rate of heat transfer coefficient in terms of the Nusselt number $\mathrm{Nu}$ at the plate is given by

$$
N u=-\left(\frac{\partial \theta}{\partial y}\right)_{y=0}=\left[\mathrm{m}_{6}\left(1-l_{3}\right)+m_{2} l_{3}\right]+\in e^{i w t}\left[\begin{array}{l}
\mathrm{m}_{8}\left(1-l_{9}\right)+m_{2}\left(l_{6}+l_{8}\right) \\
+m_{6} l_{5}+m_{4} l_{7}
\end{array}\right]
$$

\section{SHERWOOD NUMBER:}

From the concentration field, the rate of mass transfer coefficient in terms of the Sherwood number $S h$ at the plate is given by

$$
S h=-\left(\frac{\partial \phi}{\partial y}\right)_{y=0}=m_{2}+\in e^{i w t}\left[m_{4}\left(1-l_{2}\right)+m_{2} l_{2}\right]
$$

\section{RESULTS AND DISCUSSION}

In the present work, we have analyzed an unsteady MHD free convection flow of radiating and reacting Jeffery fluid past a vertical porous plate in slip-flow regime with heat source. Final results are computed for variety of physical parameters which are presented by means of graphs. The results are obtained to illustrate the influence of Jeffrey parameter $\lambda_{1}$, thermal Grashof number $G r$, Solutal Grashof number $G m$, magnetic field parameter M, Schmidt number Sc heat absorption parameter Qo absorption of radiation parameter $\mathrm{Q}_{1}$ and chemical reaction parameter $\gamma$ on the velocity, temperature and the concentration profiles. The values of some of the physical parameters are taken as constant such as $\lambda_{1}=2, M=3, G r=4, G m=2, \operatorname{Pr}=0.7$, $\gamma=0.5, \mathrm{Qo}=1, \mathrm{Q}_{1}=1, S c=0.6$ and $K_{2}=0.5$ in all the figures. We discussed skin friction coefficient, Nusselt number and Sherwood number by fixing various values on above mentioned parameters involved in the problem.

Fig. 2 describes the effects of Jeffrey parameter $\lambda_{1}$ on the velocity distribution. As we observe that the velocity decrease with an increase in $\lambda_{1}$. It is observed that it satisfies the physical nature of the fluid flow, if we increase the Jeffrey parameter then the viscosity of the fluid is enhanced and the velocity of the fluid will decrease automatically. Also, we observe that the velocity is more effect in slip flow when compare to velocity without slip flow. Fig. 3 reveals the effect of magnetic parameter $M$ on the velocity distribution. As expected, velocity is observed to decrease with an increase in $M$ because the presence of magnetic field in an electrically conducting fluid introduces a force called the Lorentz force, which acts against the flow if the magnetic field is applied in the normal. As in the present problem this type of resisting force slows down the fluid velocity as shown in this figure. The effect of increasing the value of the heat source parameter $Q_{0}$ is to decrease the boundary layer as shown in Fig. 4 which expected due to the fact that when heat is absorbed the buoyancy force decreases which retards the flow rate and thereby giving rise to decrease in the velocity profiles. The opposite trend is observed for the case of increasing the value of the porous medium parameter, the velocity is also increased as shown in Fig. 5. The opposite trend is observed for the case of increasing the value of the slip parameter $h$, the velocity is also increased as shown in Fig. 6.

It is observed from Fig. 7, that an increase in Grashof number $G r$ for heat transfer leads to a rise in the values of velocity, due to enhancement in buoyancy force with or without slip parameter $h$. To this effect, at higher Grashof number $G r$ the flow at the boundary is turbulent while at lower $G r$ the flow at the boundary is laminar. A similar effect is noticed from Fig. 8, in the presence of modified
Grashof number $G m$, which also increases fluid velocity as increase in $G r$. From the Fig. 9 it is observed that the velocity increases with an decrease in Prandtl number $P r$ with or without slip parameter. Increasing $P r$ clearly reduces the motion of the fluid. This is because Prandtl number is defined as the ratio of momentum diffusivity (viscous force) to thermal diffusivity. The increase of Pr indicates increase in the viscosity of the fluid which makes the fluid thick. Here also, we observe from Fig. 10 that the velocity increases with an increasing of Schmidt number in with or without slip flow.

Fig. 11 represents graph of temperature distribution for different values of heat source parameter, Prandtl number and radiation parameter. Here we observe that the temperature decreases with an increase in the heat absorption parameter, because when heat is absorbed, the buoyancy force decreases the temperature profile and the temperature decreases with an increase of Prandtl number, it is obvious that the temperature profile is reduced as $P r$ increase in the boundary layer. This is because larger Pr amounts to lesser thermal conductivity. Hence Pr can be used to rise the rate of cooling in conducting flows. And also, we noticed that increase in the radiation parameter decreases the temperature distribution in the thermal boundary layer due to decrease in the thickness of the thermal boundary layer with thermal radiation parameter $\mathrm{R}$

Fig. 12 depicts the graph of temperature profiles for various values of absorption of radiation parameter $\mathrm{Q}_{1}$. It is seen that the effect of absorption of radiation parameter is to increase temperature in the boundary layer as the radiated heat is absorbed by the fluid which is responsible for increase in the temperature of the fluid very close to the porous boundary layer and its effect diminished far away from the porous boundary. The effect of chemical reaction parameter on concentration is shown in Fig. 13. It is observed that for increasing value of chemical reaction parameter concentration decreases. This is due to the fact that destructive chemical reaction reduces the solutal boundary layer thickness and increases the mass transfer. Similar facts are seen in the case when Schmidt number is increased as noted in same figure. It may also be observed that an increase in the Schmidt number suppresses the concentration boundary layer thickness which is associated with the reduction in the concentration. Physically, the increase of Sc means decrease of molecular diffusion. Hence, the concentration of the species is higher for small values of Sc and lower for larger values of Sc.

Now to discuss the other physical quantities like skin-friction coefficient, local Nusselt number and Sherwood number, computations are carried out and the results are presented in Figs. 14-18. Fig. 14, describes the skin-friction $\tau$ vary with Jeffrey parameter $\lambda_{1}$ for different values of Prandtl number $\operatorname{Pr}$, heat absorption $Q_{0}$, radiation parameter $R$ and heat source parameter $Q_{1}$. It is observed that the skin friction $\tau$ is higher at $\lambda_{1}=2$ and decreases were as increasing the Jeffrey parameter $\lambda_{1}$ for different values of $\operatorname{Pr}, Q_{0}, R$ and $Q_{1}$. Fig. 15, depicts the skinfriction vary with Jeffrey parameter $\lambda_{1}$ for different parameters $G r, G m$ and $S c$. Here we observe that the Skin-friction attains the maximum value at $\lambda_{1}=2$ and decreases were as increasing in the Jeffrey parameter $\lambda_{1}$ for different values of $G r, G m, S c$. The effects of Skin-friction $\tau$ vary as magnetic parameter $M$ is shown in Fig. 16, for different values of $\lambda_{1}, k_{2}$ and $\gamma$. It is observed that, Skin-friction $\tau$ attains the maximum value at $M=2$ and decreases were as increasing in magnetic parameter $M$ for different values of $\lambda_{1}, k_{2}$ and $\gamma$.

Fig. 17 shows the effects of Nusselt number vary with Prandtl number for different parameters $Q_{0}, Q_{1}$ and $R$, from which it is observe that the Nusselt number attains the maximum value at minimum value of $\operatorname{Pr}=0$ and it is decreases were as an increasing of Prandtl number Pr for different values of $Q_{0}, Q_{1}$ and $R$. The effects of Sherwood 
number vary with chemical reaction parameter $\gamma$ for different parameters $S c, t, \omega$ as shown in from Fig. 18. It is noticed that the Sherewod number attains the maximum value at $\gamma=0$ and decreasing were as increasing of the chemical reaction parameter for different values of $S c, t$ and $\omega$.

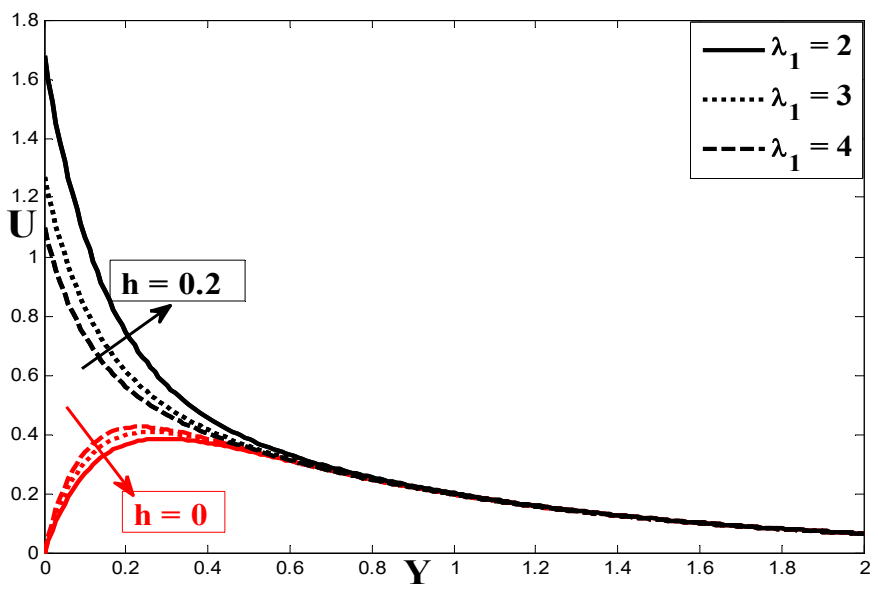

Fig. 2 Velocity profiles for different values of $\lambda_{1}$ with or without slip

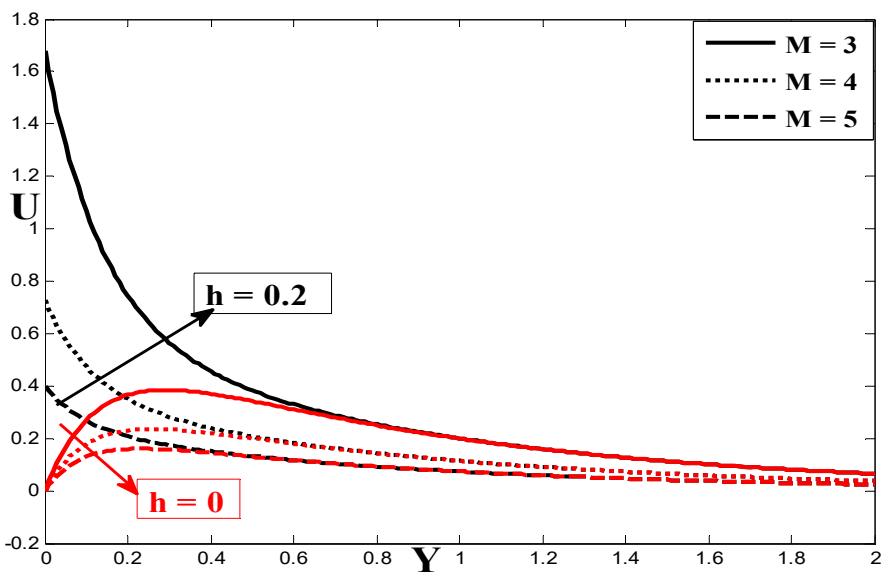

Fig. 3 Velocity profiles for different values $M$ with or without slip

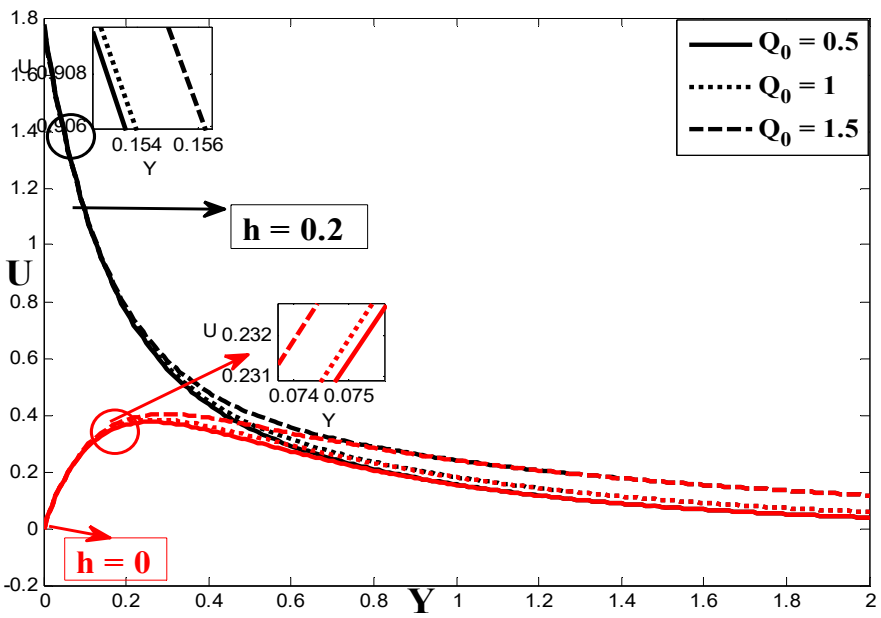

Fig. 4 Velocity profiles for different values of $Q_{0}$ with or without slip

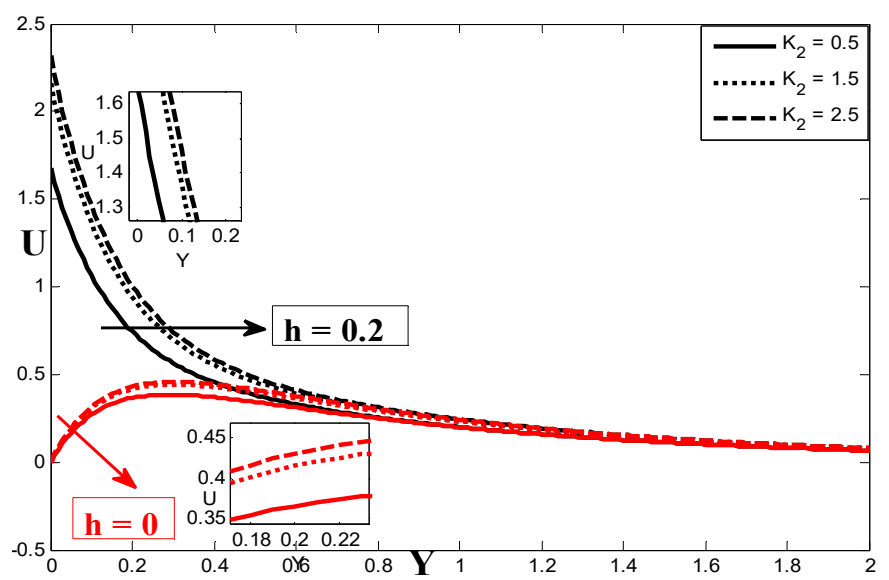

Fig. 5 Velocity profiles for different values of $k_{2}$ with or without slip

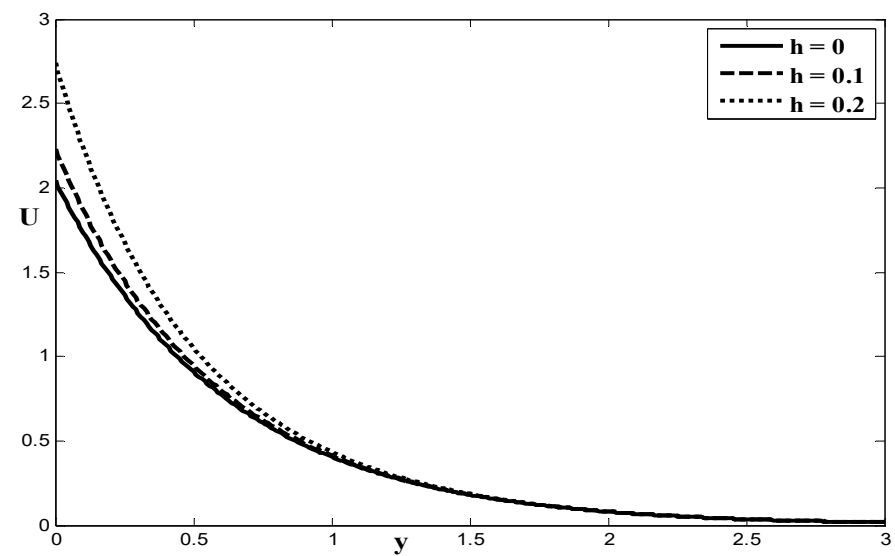

Fig. 6 Velocity profiles for different values of Slip parameter

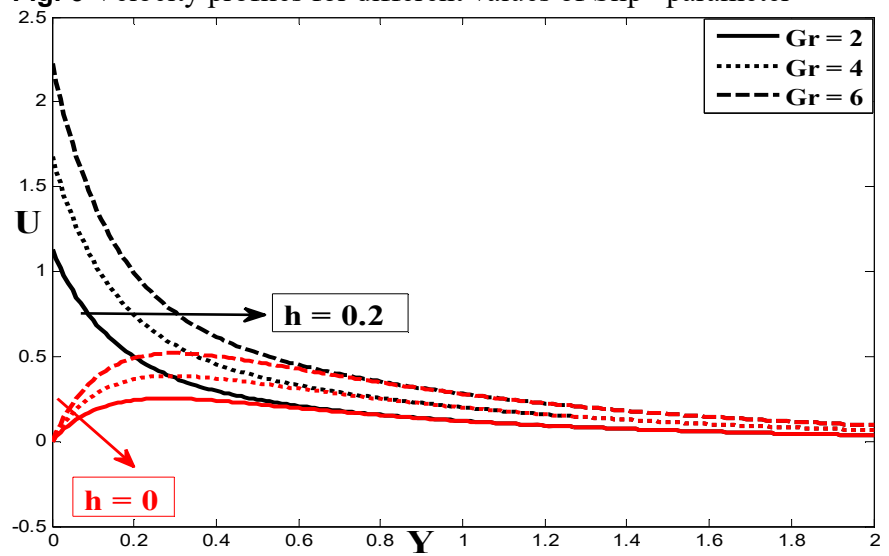

Fig. 7 Velocity profiles for different values of $G r$ with or without slip

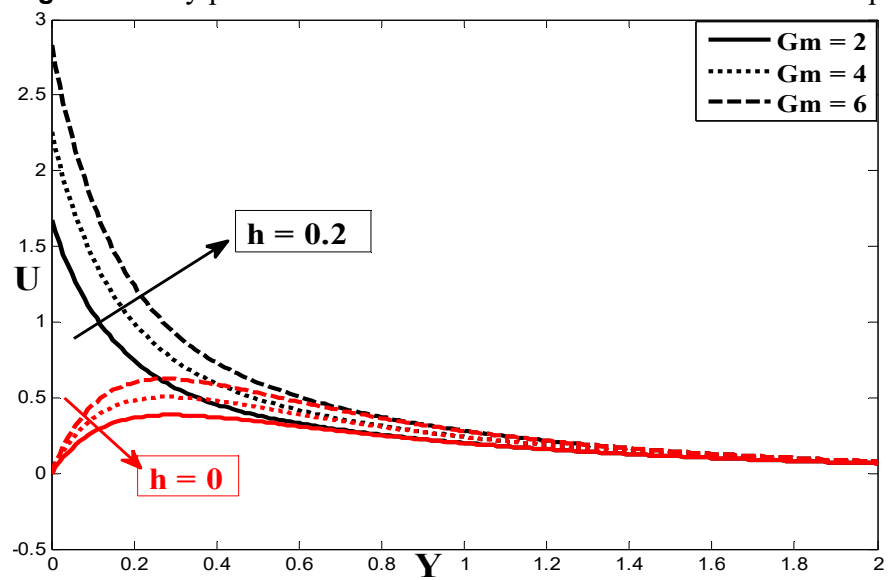

Fig. 8 Velocity profiles for different values of $G m$ with or without slip 


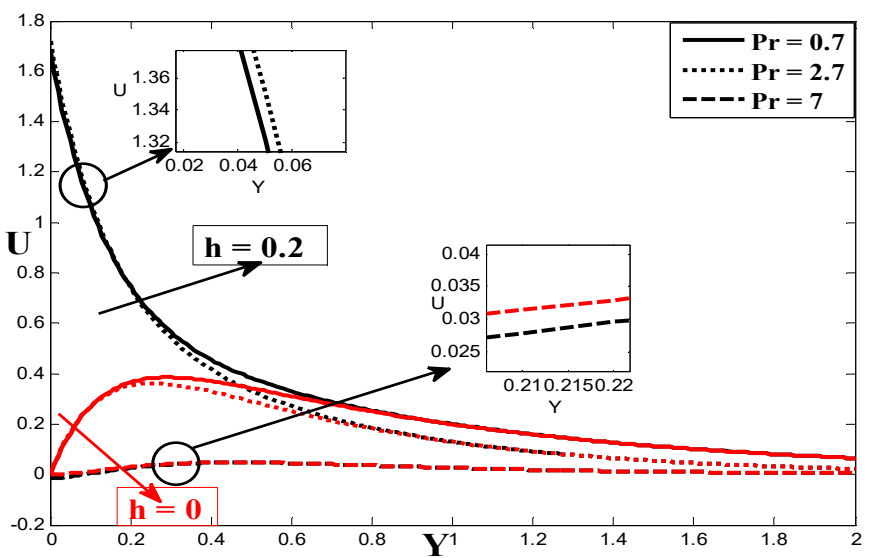

Fig. 9 Velocity profiles for different values of Pr with or without slip

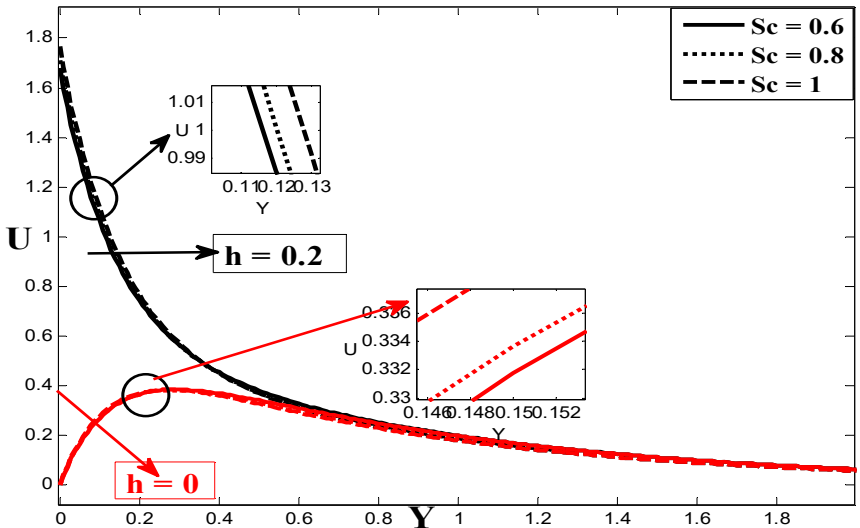

Fig. 10 Velocity profiles for different values of $S c$ with or without slip

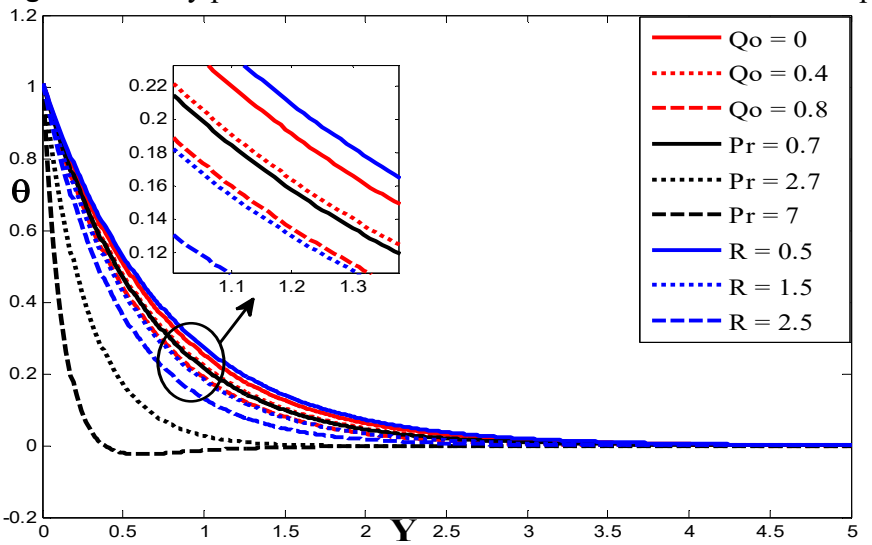

Fig. 11 Temperature profile for different values of $Q_{0}$, Pr and R .

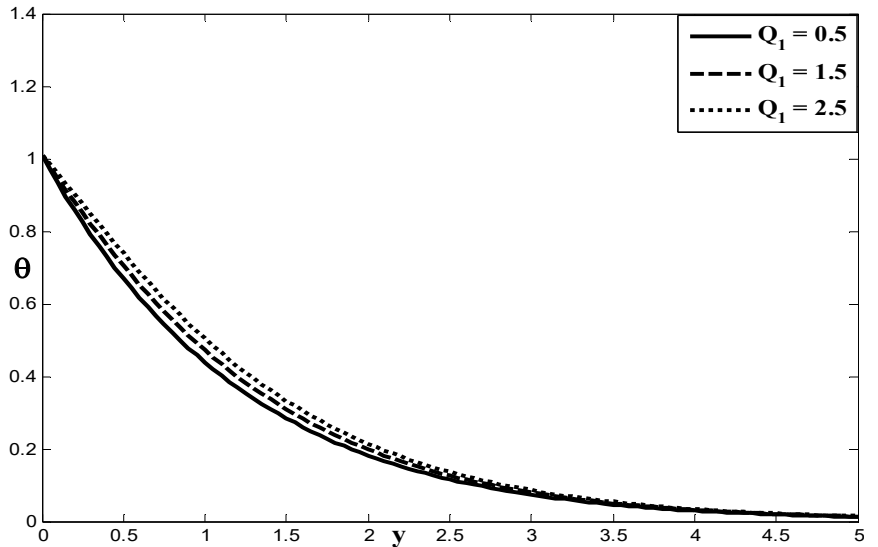

Fig. 12 Temperature profile for different values of $Q_{1}$.

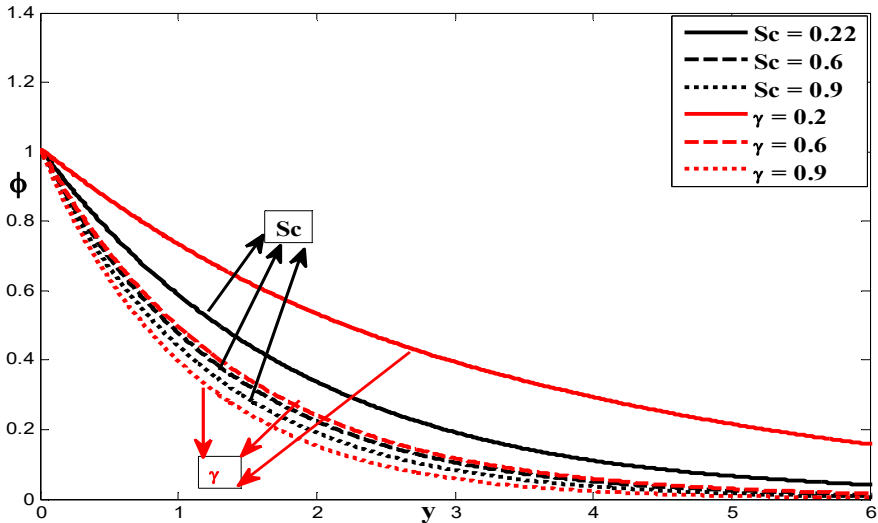

Fig. 13 Concentration profile for different values of $S c$ and $\gamma$.

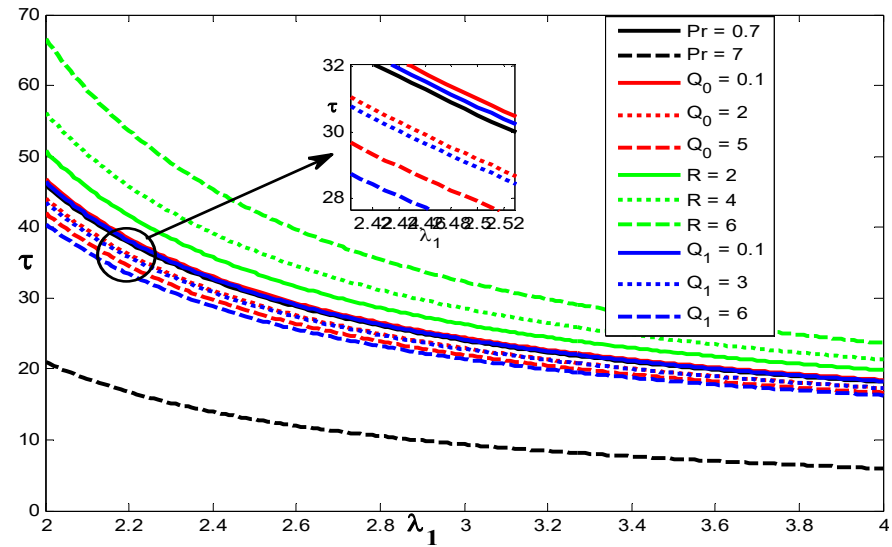

Fig. 14 The Skin-friction $\tau$ profile varies as Jeffrey parameter for different values of $\operatorname{Pr}, Q_{0}, R$ and $Q_{1}$

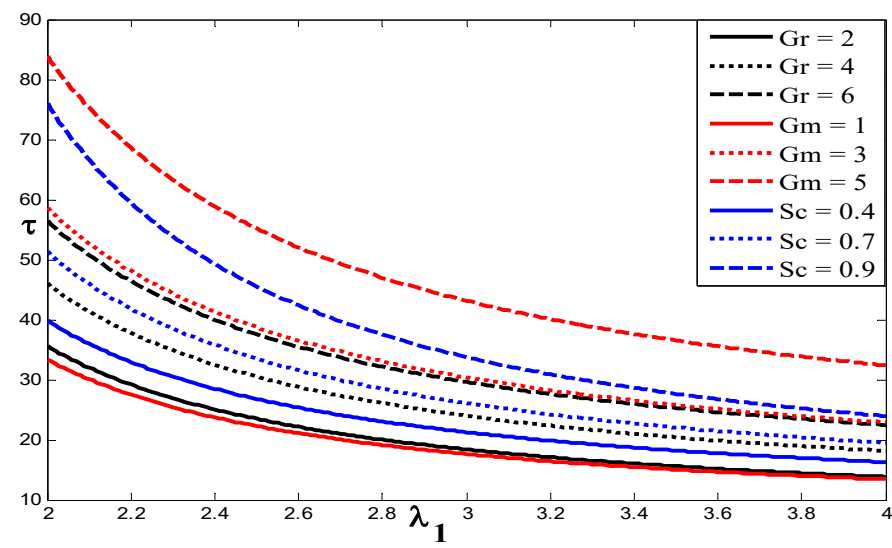

Fig. 15 The Skin-friction $\tau$ profile varies as Jeffrey parameter $\lambda_{1}$ for different values of $G r, G m$ and $S c$.

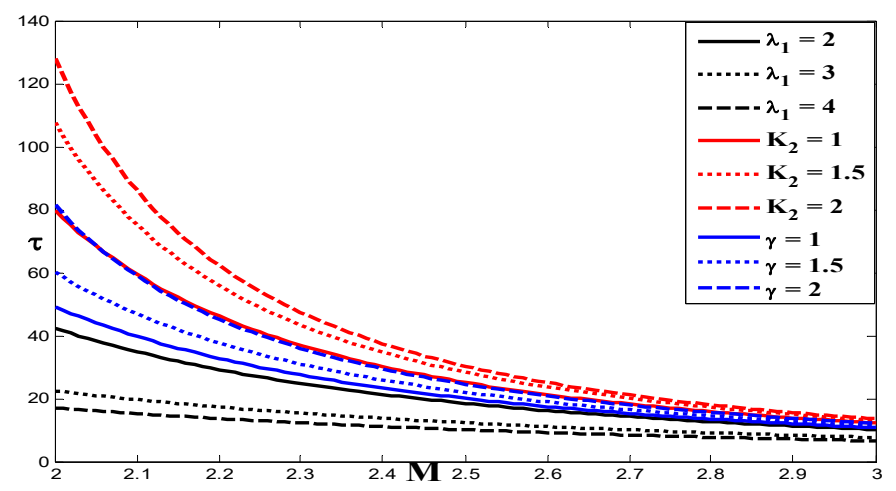

Fig. 16 The Skin-friction $\tau$ profile varies as Magnetic parameter $M$ for different values of $\lambda_{1}, \mathrm{~K}_{2}$ and $\gamma$. 


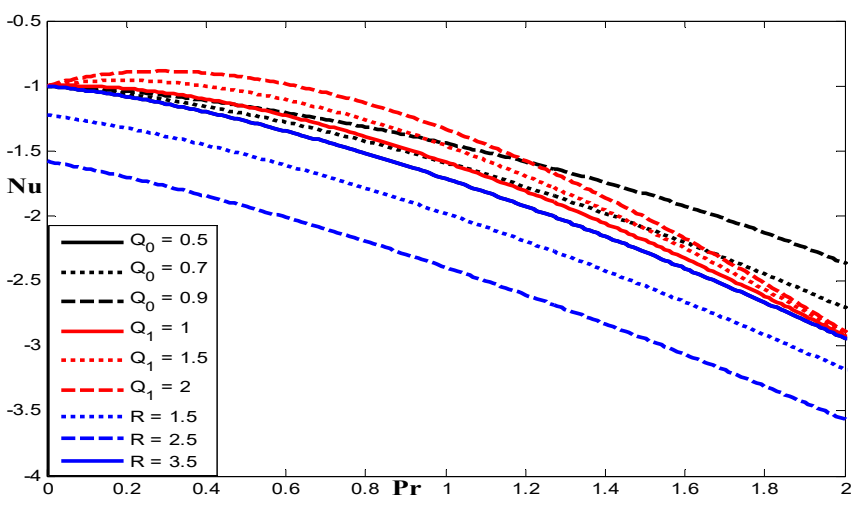

Fig. 17 The Nusselt number (Heat transfer) profile varies as Prandtl number for different values of $Q_{0}, \mathrm{Q}_{1}$ and $R$

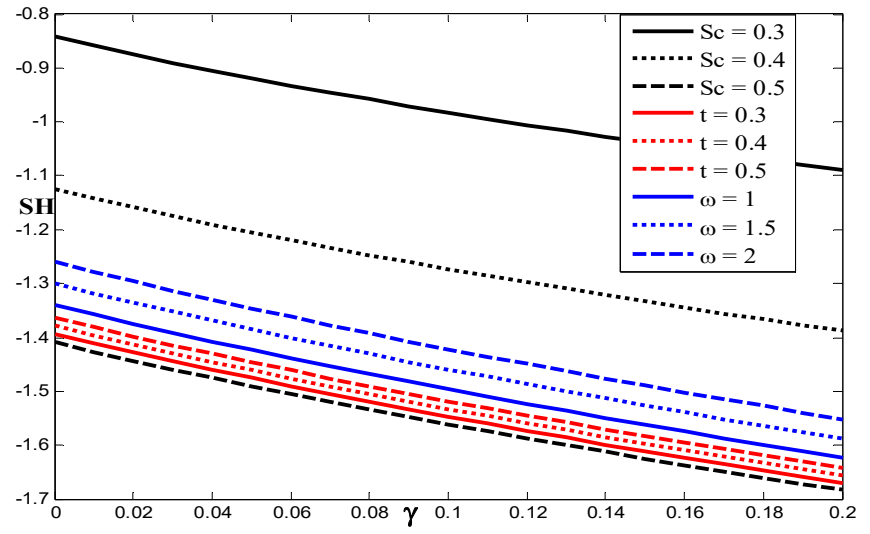

Fig. 18 The Sherwood number (mass transfer) profile varies as chemical reaction parameter $\gamma$ for the different values of $S c, t$ and $\omega$.

\section{CONCLUSIONS}

The governing equations for unsteady MHD free convective heat and mass transfer flow of radiating and reacting fluid past a vertical porous plate considering the effect of slip conditions were formulated. The resulting partial differential equations were transformed into a set of ordinary differential equations using two-term series and solved in closed-form. In the absence of chemical reaction, our results are found in good agreements with the results of Sharma and Chaudhary (2003). The conclusions of the study are as follows:

1) Velocity increases significantly with increasing values of modified Grashoof number.

2) Velocity and skin-friction coefficient increase for generative chemical reactions. However, destructive chemical reactions have opposite effect on velocity and skin-friction coefficient.

3) Velocity decreases and Nusselt number increases with the increase of radiation parameter.

4) Sherwood number increases and concentration decreases for destructive chemical reactions. However, generative chemical reactions have opposite effect on Sherwood number and concentration.

\section{NOMENCLATURE:}

$A \quad$ Amplitude of suction velocity

$B_{0} \quad$ Magnetic field strength

C* Concentration

$C_{p} \quad$ Specific heat at constant pressure

$C_{f x} \quad$ Skin friction co-efficient

$D \quad$ Mass diffusion co-efficient

$R \quad$ Radiation parameter

$G \quad$ Acceleration due to gravity

Gr Thermal Grashof number

Gm Solutal Grashof number
$G$

$K_{I} \quad$ Permeability parameter

$K_{2} \quad$ Rotation parameter

$\Gamma \quad$ Chemical reaction parameter

$M \quad$ Hatmann number (Magnetic field parameter)

$N \quad$ Dimension less material parameter

$n \quad$ Dimension less exponential index

$K_{c} \quad$ Dimensionless Chemical reaction parameter

$\mathrm{Nu} \quad$ Nusselt number

Pr Prandtl number

$Q_{0} \quad$ Heat absorption co-efficient

$R_{x} \quad$ Local Reynolds number

Sc Schmidt number

Sh Sherwood number

$T \quad$ Temperature

$t^{*} \quad$ Dimensional time

$\lambda_{1} \quad$ Jeffrey parameter

So $\quad$ Soret Number

$a^{*} \quad$ Mean absorption coefficient

T* Temperature

$Q_{1} \quad$ Heat source parameter

$\theta \quad$ Dimension less temperature

$\phi \quad$ Dimension less Concentration

$U_{0} \quad$ Scale of free stream velocity

$u^{*}, v * \quad$ Dimensional velocity components

$V_{0} \quad$ Scale of suction velocity

$x^{*}, y^{*} \quad$ Dimensional co ordinate system

$x, y \quad$ Distance along and perpendicular to the plate respectively

$h \quad$ Slip parameter

Greek Symbols

$\chi \quad$ Dimension less material parameter

$\beta_{c} \quad$ Coefficient of volumetric concentration expansion

$\beta_{T} \quad$ Coefficient of volumetric thermal expansion

$\varepsilon \quad$ Scalar constant

$\Phi$ Dimensionless heat absorption coefficient

$\kappa \quad$ Thermal conductivity

$\sigma \quad$ Electrical conductivity

$\rho \quad$ Density of the fluid

$\eta \quad$ Dimensionless normal distance

Subscripts and Superscripts

* Dimensional properties

$P \quad$ Plate

$W \quad$ Wall condition

$\infty \quad$ Free stream condition

v $\quad$ Kinematic viscousity

$v_{0}^{*} \quad$ Mean suction velocity,

$\omega^{*} \quad$ Frequency of oscillation

\section{REFERENCES:}

Anjali Devi, S.P., and Raj, J. W. S., 2011, "Thermo Diffusion Effects on Unsteady Hydro Magnetic Free Convection Flow with Heat and Mass Transfer Past a Moving Vertical Plate With Time Dependent Suction And Heat Source In A Slip Flow Regime," Int. J. of Appl. Math and Mech., 7, 20-51.

Bird, R.B., Armstrong, R.C. and Hasager, O., 1987 "Dynamics of Polymeric Liquids," Fluid dynamics, vol. 1., Wiley;

Das, K., Acharya, N., and Kundu, P.K., 2015, “ Radiative Flow of MHD Jeffrey Fluid Past a Stretching Sheet with Surface Slip and Melting Heat Transfer," Alex. Eng. J., 54, 815-821.

http://dx.doi.org/10.1016/j.aej.2015.06.008

Hayat, T., Khan, M., Fakhar, K., and Amin, N., 2010, "Oscillatory Rotating Flows of a Fractional Jeffrey Fluid Filling a Porous Space," 
J. Porous Media, 13, 29-38.

http://dx.doi.org/10.1615/JPorMedia.v13.i1.30

Hayat, T., Mustafa, M. and Naturforsch, Z., 2010, "The Effect of Thermal Radiation on the Unsteady Mixed Convection Flow of a Jeffrey Fluid Past a Porous Vertical Stretching Surface Using Homotopy Analysis Method (HAM)," 65a, 711-719.

Hayat, T., Shehzad, S.A., Qasim, M.,and Obaidat, S., 2012, “ Radiative Flow of Jeffery Fluid in a Porous Medium with Power Law Heat Flux and Heat Source," Nucl. Eng, 243, 15-19.

https://doi.org/10.1016/j.nucengdes.2011.11.005

Hussain, T., Shehzad, S.A., Hayat, T., Alsaedi, A., Al-Solamy, F. and Ramzan, M., 2014, "Radiative Hydromagnetic Flow of Jeffrey Nanofluid by an Exponentially Stretching Sheet," Plos One, 9(8), 1-9. http://dx.doi.org /10.1371/journal.pone.0103719

Idows, A.S., Joeph, K. M. and Daniel, S., "Effect of Heat and Mass Transfer on Unsteady MHD Oscillatory Flow of Jeffrey Fluid in a Horizontal Channel with Chemical Reaction," IOSR Journal of Mathematics, 8(5), 74-87. www.iosrjournals.org.

Jeffery, H., 1929, The earth. 4th ed. London: Cambridge University Press.

Khan. M., 2007, "Partial Slip Effects on the Oscillatory Flows of a Fractional Jeffrey Fluid in a Porous Medium," J. Porous Media, 10,473-487.

http://dx.doi.org/10.1615/JPorMedia.v10.i5.50

Kim. J Youn., 2000, "Unsteady MHD convective heat transfer past a semi-infinite vertical porous moving plate with variable suction," International Journal of Engineering Sciences, 38(8), 833-845. https://doi.org/10.1016/S0020-7225(99)00063-4

Lakshminarayana, P., Sreenadh, S., Sucharitha, G., and Nandgopal, K., 2015, "Effect of Slip and Heat Transfer on Peristaltic Transport of a Jeffrey Fluid in a Vertical Asymmetric Porous Channel," Adv. Appl. Sci. Res., 6(2), 107-118.

www.pelagiaresearchlibrary.com

Michael, J., Martin and lain, and Boyd, D., 2001, "Blasius Boundary Solution with Slip Flow Conditions,"

http://doi.org/10.1063/1.1407604.

Mittal, P.K., Bijalwan,M., 2005, “A study of vorticity of fluctuating flow of an visco-elastic fluid past an infinite plate with variable suction in slip flow regime," Acta Ciencia Indica XXXIV M, 3, 903.

Muhammad Saqib., Farhad Ali., Ilyas Khan, Nadeem Ahmad Sheikh, Syed Aftab Alam Jan and Samiulhaq., "Exact Solutions for Free Convection Flow of Generalized Jeffrey Fluid: A Caputo-Fabrizio Fractional Model,"

http://dx.doi.org/10.1016/j.aej.2017.03.017

Nazibuddin Ahmed., and Kishor Kumar Das., 2013, "MHD Mass Transfer Flow Past a Vertical Porous Plate Embedded in a Porous Medium in a Slip Flow Regime with Thermal Radiation and Chemical Reaction", Open Journal of Fluid Dynamics, 3, 230-239. http://dx.doi.org/10.4236/ojfd.2013.33028

Pal, D., Talukdar, B., 2010, "Perturbation Analysis of Unsteady Magneto Hydrodynamic Convective Heat and Mass Transfer in a Boundary Layer Slip Flow Past a Vertical Permeable Plate with Thermal Radiation and Chemical Reaction," Communications in Nonlinear Science and Numerical Simulation, 15(7), 1813-1810.

https://doi.org/10.1016/j.cnsns.2009.07.011
Rajput,U.S., Varshney N.K., and Rajput.D.,2008, "Free Convection Flow of Magneto Polar Fluid Through Porous Medium in Slip Flow Regime with Mass Transfer," Acta Ciencia Indica XXXIV M.

Ramesh Babu, K., Parandhama, A., Venkateswara Raju, K., Raju, M.C., and Satya Narayana, P.V., 2017, "Unsteady MHD Free Convective Flow of a Visco-Elastic Fluid Past an Infinite Vertical Porous Moving Plate with Variable Temperature and Concentration," Int. J. Appl. Comput. Math.

http://dx.doi.org/10.1007/s40819-017-0306-8.

Rudraiah, N., Dulal Pal, and Sivakumar, P. N., 1988 "Effects of Slip and Magnetic Field on Composite System," Fluid Dynamic. Res., 4, 255-270.

Santhosh, N., and Radhakrishnamacharya, D., 2014, "Jeffrey Fluid Flow Through Porous Medium in the Presence of Magnetic Field in Narrow Tubes. Hindawi Publishing Corporation," International Journal of Engineering Mathematics Article, 1-8.

$\underline{\text { http://dx.doi.org/10.1155/2014/713831 }}$

Sharma, P. K., and Chaudhary, R. C., 2003, "Effect of Variable Suction on Transient Free Convection Viscous Incompressible Flow Past a Vertical Plate with Periodic Temperature Variations in SlipFlow Regime," Emirates, J. Engineering Research, 8, 33-38.

Shehzad, S.A., Alsaedi, A., and Hayat, T., 2013, "Influence of Thermophoresis and Joule Heating on the Radiative Flow of Jeffrey Fluid with Mixed Convection” ,Braz. J. Chem. Eng., 30(4),897-908. www.abeq.org.br/bjche

Shehzad, S.A., Hayat, T., Alhuthali, M.S. and Asghar, S., 2014, "MHD Three-Dimensional Flow of Jeffrey Fluid with Newtonian Heating," J. Cent. South Univ., 21, 1428-1433.

http://dx.doi.org/10.1007/s11771-014-2081-6

Shojaeian, M. and Koşar, A., 2014, "Convective Heat Transfer and Entropy Generation Analysis on Newtonian and Non-Newtonian Fluid Flows Between Parallel-Plates Under Slip Boundary Conditions," Int. J. Heat Mass Transfer, 70, 664-673.

https://doi.org/10.1016/j.ijheatmasstransfer.2013.11.020

Sreenadh, S., Prakash, J., Parandhama, A. and Ravi Kumar, Y.V.K., 2014, "Oscillatory Flow of a Conducting Jeffrey Fluid in a Composite Porous Medium Channel," 10th International Conference on Heat Transfer, Fluid Mechanics and Thermodynamics, 14-16 July2014, Florida. 2350-2358.

Sreenadh, S., Rashidi, M.M., Kumara Swamy Naidu, K. and Parandhama, A.,2016, "Free Convection Flow of a Jeffrey Fluid Through a Vertical Deformable Porous Stratum," Journal of Applied Fluid Mechanics, 9(5), 2391-2401.

http://dx.doi.org/10.18869/acadpub.jafm.68.236.25549

Vajravelu, K., Sreenadh, S., Lakshminarayana, P., and Sucharitha, G., 2008, "The Effect of Heat Transfer on the Nonlinear Peristaltic Transport of a Jeffery Fluid Through a Finite Vertical Porous Channel," Int. J. Bio math, 9(2), 1650023, 24 pages. https://doi.org/10.1142/S1793524516500236 